\title{
Inelastic UV Scattering in a Floating Water Bridge
}

\author{
Elmar C. Fuchs ${ }^{1}$, Brigitte Bitschnau ${ }^{2}$, Silvia Di Fonzo ${ }^{3}$, Alessandro Gessini ${ }^{3}$, Jakob Woisetschläger ${ }^{4}$ and Filippo \\ Bencivenga $^{3}$ \\ 1. Wetsus, Centre of Excellence for Sustainable Water Technology, Agora 1, 8900 CC Leeuwarden, The Netherlands \\ 2. Institute of Physical and Theoretical Chemistry, Graz University of Technology, Stremayrgasse 9/I (A), 8010 Graz, Austria \\ 3. Sincrotrone Trieste S.C.p.A. di interesse nazionale, Strada Statale 14 - km 163,5 in AREA Science Park, 34012 Basovizza, Trieste, \\ Italy \\ 4. Institute for Thermal Turbomachinery and Machine Dynamics, Graz University of Technology, Inffeldgasse 25 A, 8010 Graz, \\ Austria
}

Received: March 25, 2011 / Accepted: Apri 1 08, 2011 / Published: August 15, 2011.

\begin{abstract}
When high voltage is applied to distilled water filled into two beakers close to each other, a watery connection forms spontaneously, giving the impression of a floating water bridge [1-8]. In this work we present the first inelastic ultraviolet scattering data of such an electrohydrodynamic bridge revealing radial gradients of Stokes- and Anti-Stokes shifts and their intensity profiles. Interpretations including density and temperature changes within the bridge are discussed. The obtained data can be satisfactorily explained by the introduction of a second phase consisting of nano bubbles. Results and interpretation are discussed in relation to similar phenomena.
\end{abstract}

Key words: Inelastic UV scattering, floating water bridge, nano bubbles.

\section{Nomenclature}

$A_{B}, A_{C}:$ scaling factors

$B$ : flat background

$c$ : sound velocity

$c_{w}$ : sound velocity of pure water at the corresponding $(\rho, T)$-value

$E$ : energy

$E_{a}$ : activation energy

$I(E)$ : scattering intensity

$I(\omega)$ : scattering intensity

$\mathrm{k}_{\mathrm{B}}$ : Boltzman constant

$L$ : vertical detector position

$M_{B} \quad$ : bulk modulus of the bridge (bubbles + water)

$M_{w}$ : bulk modulus of water

$Q$ : wavevector length

$\Delta Q$ : finite difference between two adjacent wave vectors

$R$ : averaged laser penetration depth

$R(E)$ : instrumental energy resolution function

$T$ : temperature (general); temperature of the bridge at the actual $R$ value

Corresponding author: Elmar C. Fuchs, Dipl.-Ing. Dr. techn., research field: electrohydrodynamic liquid bridging. E-mail: elmar.fuchs@wetsus.nl.
$T_{0}: 46.1^{\circ} \mathrm{C}$ (temperature of the bridge surface)

$\Delta T: T_{0}-T$ (difference between the outside bridge temperature and the temperature at the actual $R$ value)

$V_{\min }$ : minimum volume allowed for the system

$X_{b u b}$ : percentage of the bubble phase

\section{Greek letters}

$\alpha$ : scattering angle

$T$ : fitting parameter for a constant radial temperature gradient

$\Delta \alpha$ : finite difference between two adjacent scattering angles

$\Gamma_{B}:$ line width of inelastic excitations

$\delta(E)$ : Dirac function

$v_{L}$ : longitudinal kinematic viscosity

$\rho$ : density of the system (at the actual $R$ value)

$\rho_{w}$ : density of pure water at the corresponding ( $\left.\rho, T\right)$-value

$\rho_{0}: 989.8 \mathrm{~kg} / \mathrm{m}^{3}$ (water density at 1 bar and $46.1{ }^{\circ} \mathrm{C}$ )

$\chi^{2}$ : chi square distribution

$\chi_{s, b u b}$ : compressibility of the bubble subsystem

$\omega$ : frequency

$\Omega_{B}$ : frequency position of inelastic excitations

\section{Operators}

$\otimes$ : convolution operator 


\section{Introduction}

In 1893, Sir William Armstrong placed a cotton thread between two wine glasses filled with chemically pure water. After applying a high voltage, a watery connection formed between the two glasses, and after some time, the cotton thread was pulled into one of the glasses, leaving, for a few seconds, a rope of water suspended between the lips of the two glasses [1]. As gimmick from early days of electricity this experiment was handed down through history until the present authors learned about it from W. Uhlig [2]. Although easy to reproduce, this watery connection between the two beakers, which is further referred to as 'floating water bridge, holds a number of interesting static and dynamic phenomena [3-8].

At macroscopic scale several of these phenomena can be explained by modern electrohydrodynamics, analyzing the motion of fluids in electric fields (e.g., the text book on Electrohydrodynamics by Castellanos [9]). On the molecular scale water can be described by quantum mechanics $[10,11]$. The gap at mesoscopic scale is bridged by a number of theories including quantum mechanical entanglement and coherent structures in water, theories which are currently discussed (e.g., Refs. [12-16] for water in general, and Ref. [17] specifically for the water bridge). Previous experiments [3] suggested a possible change of the water micro structure inside the water bridge; first neutron scattering experiments [5] showed no difference in the microdensity of a $\mathrm{D}_{2} \mathrm{O}$ bridge compared to the bulk; recent $2 \mathrm{D}$ neutron scattering experiments [6] indicated a preferred molecular orientation within a floating heavy water bridge; detailed optical investigations [7] suggested the existence of a mesoscopic bubble network within the water bridge, a hypothesis supported by neutron scattering [5] and quantum field theory [17]. A Raman scattering study on vertical water bridges reported on a polarized water structure induced by the electric field [18]. First studies of the electrochemistry in a water bridge set-up of inorganic [19] and organic [20] solutions revealed bridge destabilization and substance transport across the bridge as well as differences in electrochemical behavior, respectively. The phenomenon seems to disappear under reduced gravity conditions [21]. As far as the basics of the water-bridge are concerned, the phenomenon is well-established in the framework of electrohydrodynamics [22] and has been described from a purely theoretical point of view $[23,24]$. According to that, the most important properties necessary for a liquid bridge formation are high dielectric permittivity, low electric conductivity and a permanent molecular dipole moment. Thus the phenomenon is not water specific but can be reproduced with any liquid of similar properties like methanol [25] or glycerol [22].

An overview is given in a recent review [25]. The properties of water at mesoscopic scales have drawn special attention due to their suggested importance to human physiology [26].

Experimentally these scales are accessible by optical measurement techniques, with most of them applicable to this experiment since the water cylinder forming between the two glass beakers floats in air freely. This is also true for the Inelastic Ultraviolet Scattering (IUVS) measurements presented in this work.

\section{Experiment}

\subsection{Instrument}

The experiment was carried out at the IUVS beamline at the ELETTRA synchrotron facility in Trieste, Italy. As light source, a frequency doubled $\mathrm{Ar}^{+}$ laser (244 nm wavelength) with a beam diameter of 0.5 $\mathrm{mm}$ was used. The radiation scattered by the sample in an almost backscattering geometry (scattering angle $2 \alpha$ $=172^{\circ}$ ) was frequency-analyzed by an $8 \mathrm{~m}$ long Czerny-Turner spectrometer able to provide a resolving power up to $10^{6}$. The $Q$-resolution of the instrument is given by $\Delta Q / Q=\cot (\alpha) \cdot \Delta \alpha$, where $\Delta \alpha$ is the angular divergence of scattered radiation. This is set by the illuminated area of the collection mirror, which is $100 \mathrm{~mm}$ in diameter and it is placed at 3,000 
$\mathrm{mm}$ distance from the sample. Therefore, $\Delta \alpha$ is about $1^{\circ}$, resulting in almost parallel rays (Fig. 1). The precision of the $Q$-resolution is therefore $\pm 0.1 \%$. The energy $(E)$ dependence of IUVS spectra $(I(E))$ was measured by a $512 \times 2048$ CCD detector $(13.5 \times 13.5$ $\mu \mathrm{m}^{2}$ pixel size) in $\omega$-range of about $1 \mathrm{THz}$ around the incoming photon frequency with an overall $\omega$-resolution of $5.6 \mathrm{GHz}$. Further details on the beamline can be found elsewhere $[27,28]$.

\subsection{Sample}

The bridge was set up as described in Ref. [3] with glass beakers inside the IUVS sample chamber. Typical integration time for acquiring a single CCD image was $900 \mathrm{~s}$. The length of the bridges investigated was $10 \mathrm{~mm}$, their diameter $\sim 2.5 \mathrm{~mm}$. The bridges showed only minor fluctuations and were stable for more than 3 hours. Since the water bridge acts as a cylindrical lens it vertically deformed its image into the $\mathrm{CCD}$, thereby increasing the apparent diameter of the bridge (the probing UV beam impinged parallel to the bridge diameter). Because the dispersive plane of the spectrometer is the horizontal one, $I(E)$ is characterized by the three vertical lines mapped onto the CCD chip
(Fig. 2), representing the inelastic scatter Stokes and anti-Stokes lines on the outside, and the elastic scatter line in the middle. In order to determine the penetration depth of the laser, ray tracing was applied on a model that assumes a bridge made up of a homogenous liquid. The vertical axis represents an average ranging from the outer edge to the different penetration depths of the laser. This is illustrated in Fig. 1. However, due to the ever-changing diameter of the bridge, the traversing density gradients [3] and its anisotropic radial density distribution [7], it is not possible to calculate precisely how deep the UV laser actually entered the bridge. We would like to point out here that Fig. 1 depicts a simplified scenario based upon the assumption of an isotropic bridge. If the deviations from that are minor, $R$ is probably representing the outer layer and an average of the whole bridge as described.

\subsection{Characterization}

In the following of the manuscript we conventionally set $R=0$ in correspondence of the vertical position $(L)$ on the CCD where we observe the rise of the inelastic scattering signal (Figs. 2 and 3), while we set $R=1$ at the bottom of the CCD image.

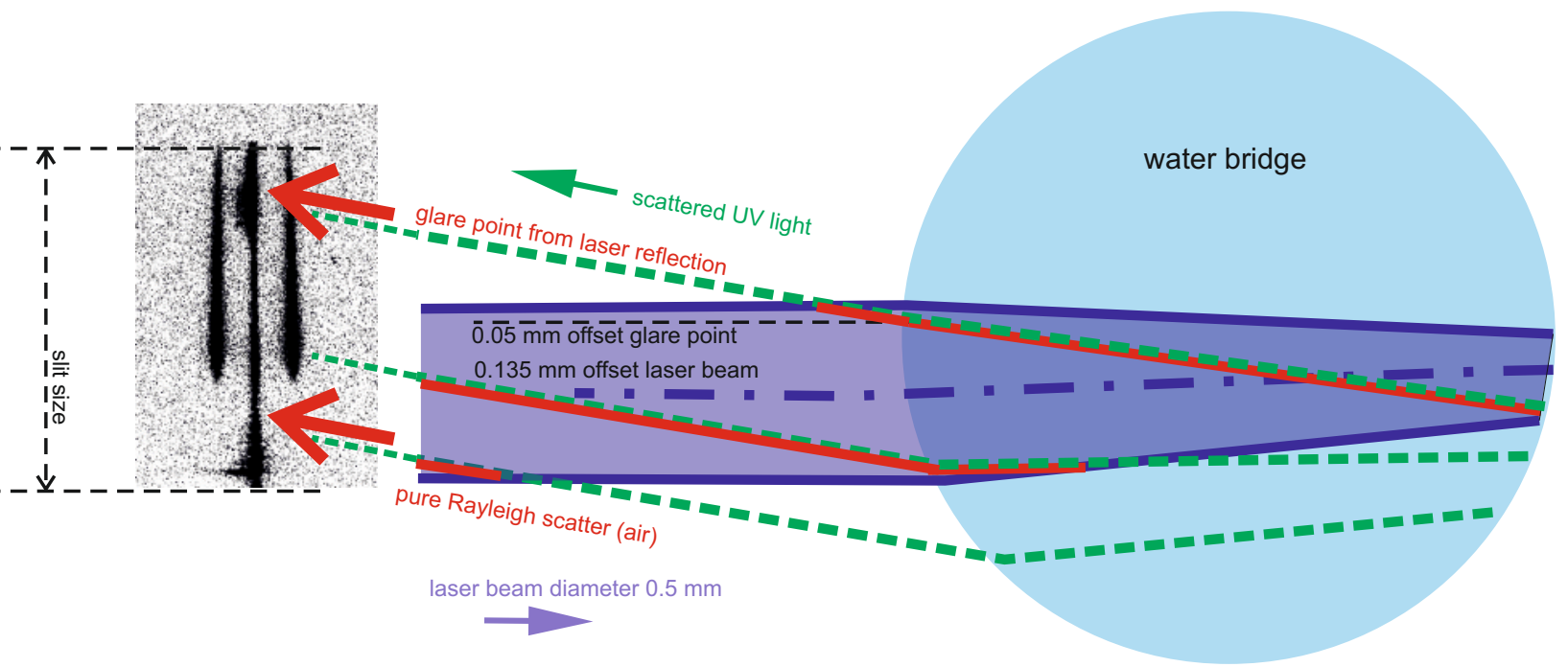

Fig. 1 Sketch of the light path of the UV laser through the water bridge simulated as isotropic water cylinder.

The blue shaded area represents the portion of the sample illuminated by the UV laser, while the green lines are some representative light paths directed to the detector, which is inclined with respect to the laser beam. The IUVS signal (showed on the left) then arises from the intersection (sketched by red lines) between illumination area and the detector deep of view. The horizontal co-ordinate $R$ on the detector starts from low penetration at $R$ close to 0 and increases to full penetration at $R \sim 0.8-1$. The beams with diminishing average (uppermost scattered beam) do not reach the detector. 


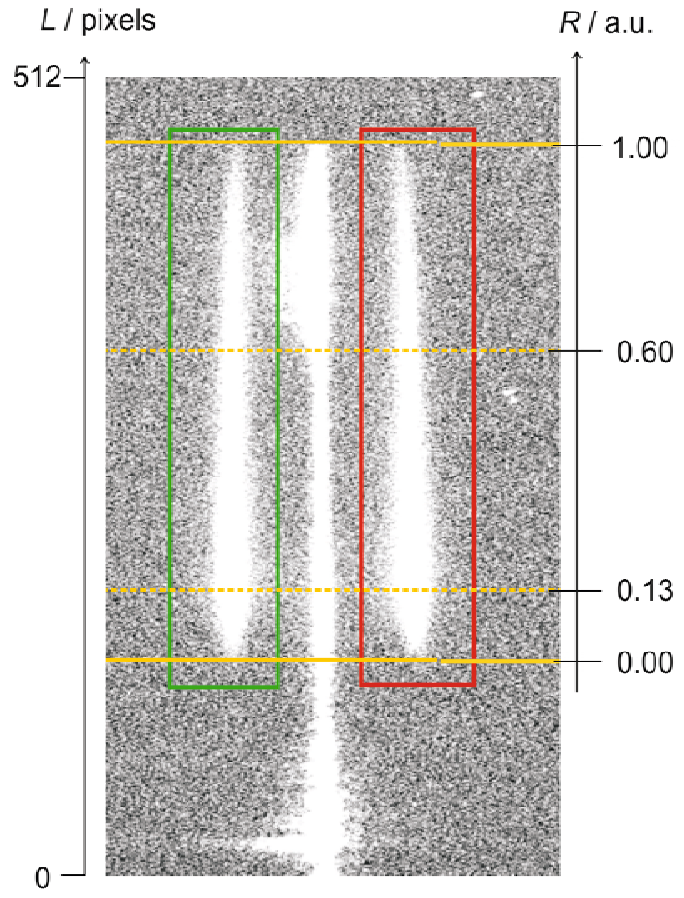

Fig. 2: CCD image of the elastic (middle) and inelastic (right and left) scattering of the laser on the floating water bridge. The red (right) and the green (left) hutched regions mark the integration area used to obtain the vertical cross sections depicted in Fig. 3. $L$ is the vertical position on the CCD, while $R$ is the penetration of the incoming beam into the bridge, conventionally set equal to zero/one in correspondence of the rise/end of inelastic signal. Additionally, $R$ positions referred to in the text are indicated.

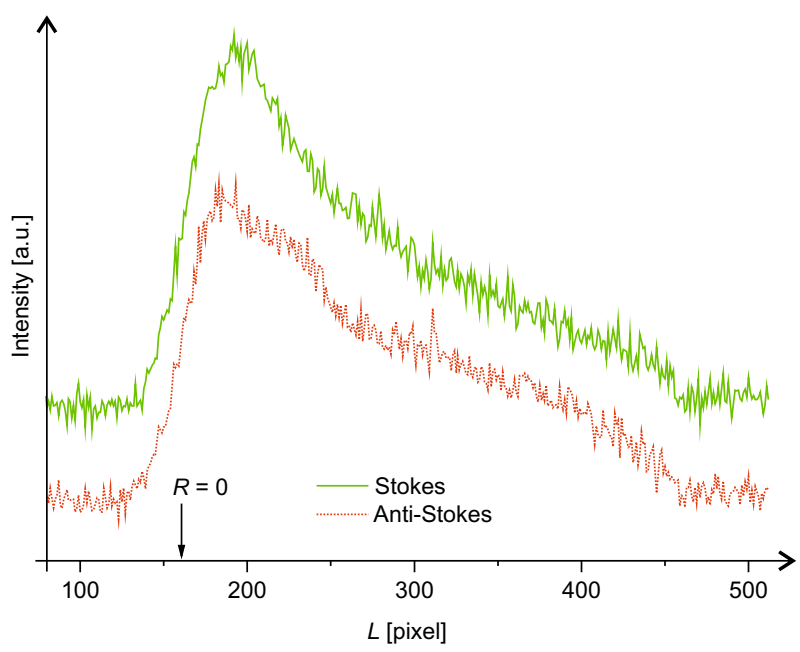

Fig. 3 Vertical cross sections of the Stokes- and Anti-Stokes lines obtained by horizontal integration of the marked areas in the CCD image (Fig. 1).

For sake of clarity the Stokes and Anti-Stokes signal are vertically shifted.
Thus $R$ values close to 0 are representative for the properties of the outer layer of the bridge; $R$ values up to $\sim 0.5$ are averaging from the edge of the bridge to the centre, and higher $R$ values average over more than half of the bridge until they cover the whole diameter at values close to 1 . Since the laser beam impinges into the bridge slightly below its center, at $R$ values below $\sim 0.5$ the leading contribution to the average arises from the outer layers, while the relative weight of the signal coming from inner core of bridge increases and may eventually become dominating only at $R$ values close to 1. Consequently, the parameter $R$ cannot be considered as an actual radius, but rather a rough measure of the extension of the experimental (almost radial) average of sample properties, which runs through a larger part of the sample for $R \sim 1$ while is limited to the external layers for $R \sim 0$.

\section{Results}

\subsection{General}

Fig. 2 shows three eye-catching features: (a) An area with Rayleigh scattering only (from $L=0$ to $R=0$ ); (b) a narrowing of the distance Stokes/Rayleigh/ Anti-Stokes line with increasing $R$; and (c) a short increase $(0<R<0.13)$ followed by a decrease $(0.13<$ $R<1)$ of the inelastic lines intensity.

Feature (a) shows the Rayleigh scattering of air only (Fig. 1, undermost ray). The high intensity at the lower end is probably due to higher orders of spurious (rainbow-) scattering from the bridge surface. For feature (b), we report some representative $I(E)$ line shapes taken at different $R$-values in Fig. 4. The resolution function of the spectrometer, shifted and scaled to one of the inelastic excitations, is shown as a dashed line. In all three cases, the broadening of the inelastic excitations is larger than the resolution line width, thus proving the significance of the measurement. It can be readily noticed that the elastic scattering contribution increases both at low and high $R$, the latter likely because of spurious scattering from 


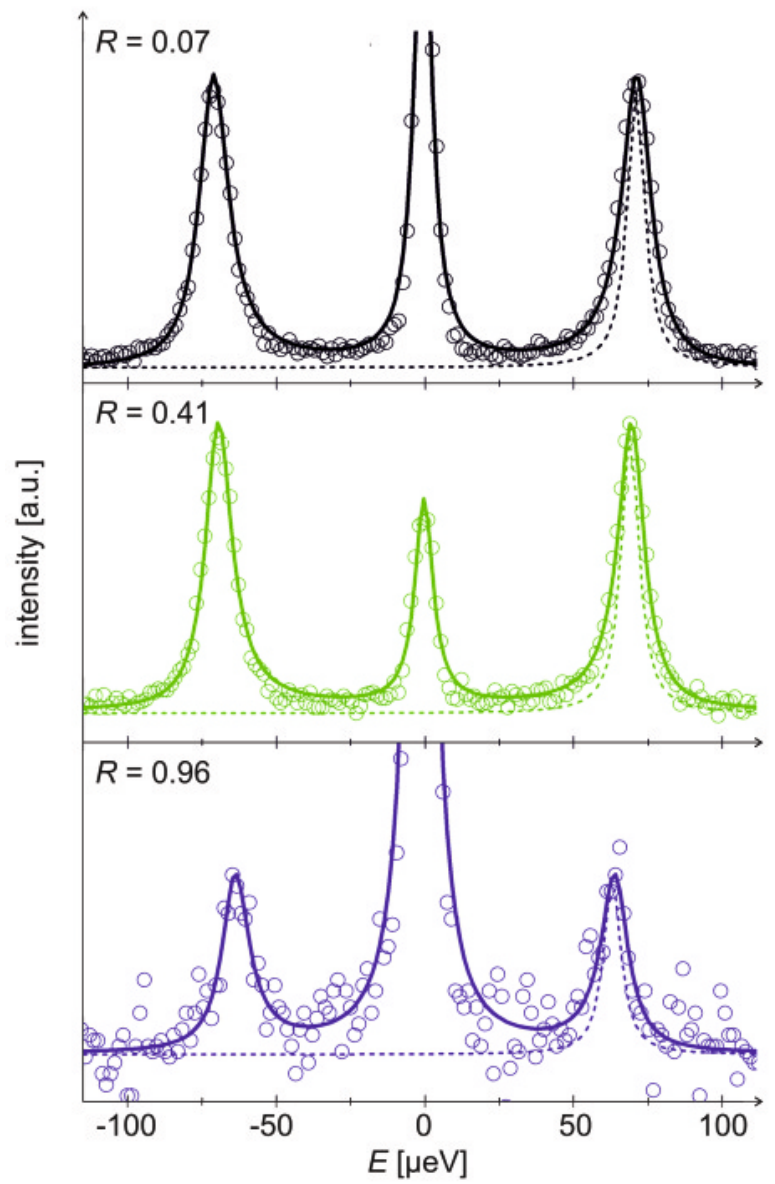

Fig. 4 Representative $I(E)$ lineshape (circles) taken at the indicated $\boldsymbol{R}$-values reported together with the corresponding best fit results (full lines).

The resolution function of the spectrometer, shifted and scaled to one of inelastic excitations, is shown as a dashed line. the bridge surfaces (glare point), while the inelastic signal first increases and then decreases with increasing $R$ (Fig. 3). The full lines in Fig. 4 are the result of the data analysis, consisting in a best fit procedure based on a standard $\chi^{2}$ minimization of the following model function:

$$
I(E)=\left[A_{B} \frac{\Omega_{B}^{2} \Gamma_{B}}{\left(E^{2}-\hbar^{2} \Omega_{B}^{2}\right)^{2}+\left(E \hbar \Gamma_{B}\right)^{2}}+A_{C} \delta(E)\right] \otimes R(E)+B
$$

where $\Omega_{B}$ and $\Gamma_{B}$ are the frequency position and line width of inelastic excitations, respectively, $A_{B}$ and $A_{C}$ are scaling factors, $\delta(E)$ is a Dirac function accounting for the elastic scattering, $R(E)$ is the instrumental energy resolution function, $\otimes$ is the convolution operator and $B$ is a flat background. The parameters $\Gamma_{B}$ and $\Omega_{B}$ can be straightforwardly associated to the longitudinal kinematic viscosity $\left(v_{L}=\Gamma_{\mathrm{B}} / Q^{2}\right)$ and sound velocity $\left(c=\Omega_{B} / Q\right)$ [28].

The inclined inelastic features of the raw data reported in Fig. 2 (feature b) and the related decrease of $\Omega_{B}$ with $R$ shown in Fig. 4 indicate a radial gradient of the average sound velocity (Fig. 5) on probing a larger portion of the bridge diameter, i.e., on increasing $R$. Such a variation of sound velocity cannot be attributed to viscoelastic effects associated to the structural relaxation process [29]. In fact, at the employed photon wavelength such a viscoelastic

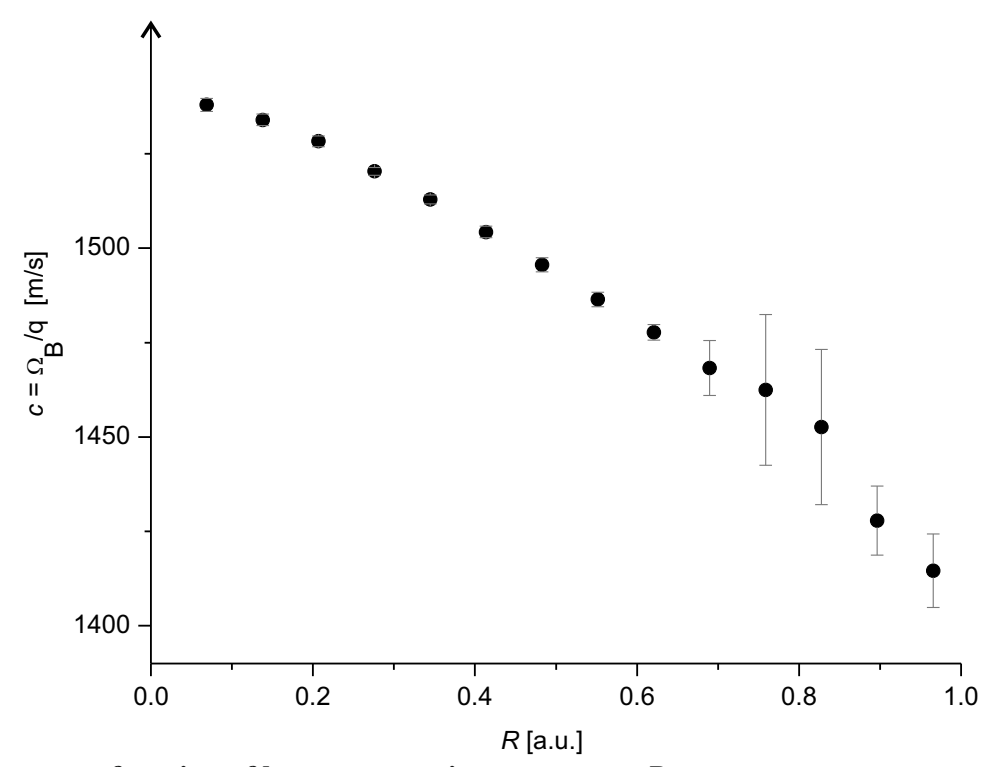

Fig. 5 Sound velocity $c$ as a function of laser penetration parameter $R$. 
variation of sound velocity is noticeable only below room temperature [30] while, as shown in the following and in agreement with thermographic results, the temperature of the water bridge is higher. As a consequence, in the following discussion any possible effect of structural relaxation is neglected, and therefore the measured $c$-values are assumed to be equal to the adiabatic sound velocity. A more thoroughly analysis of the structural relaxation phenomenology will be the subject of further investigations to be carried out at shorter photon wavelength, where appreciable viscoelastic effects are expected [30].

\subsection{Temperature and Density}

Since $R$ is an average value an intensity increase of the inelastic lines would be expected, starting with a few scatterers at $R=0$ (penetration depths some tens of microns) and exciting all scatterers at $R=1$ (penetration depth $25 \mathrm{~mm}$-the whole bridge). Both this behavior and its opposite are observed (feature $\mathrm{c}$ ): From $R=0$ to $R=0.13$, the inelastic peak intensity increases, as expected, while from $R=0.13$ to $R=1$ it decreases again. A possible explanation for this seeming contradiction is a temperature decrease inside of the bridge, since the intensity is proportional to $\mathrm{k}_{\mathrm{B}} T$. The gradient of sound velocity can also be associated with a temperature $(T)$ variation. Whereas in the present case the sound velocity data at $R \sim 0$ indicates that the local outside temperature of the bridge is $46^{\circ} \mathrm{C}$, in reasonable agreement with recent thermographic measurements [7], at $R \sim 1$ the sound velocity datum indicates an average bridge temperature of about $0{ }^{\circ} \mathrm{C}$, which would require a frozen or supercooled bridge core. Due to the variety of dynamics observed within the bridge [4, 7], this seems unlikely, unless it is not considered as actual temperature decrease in terms of thermal energy decline, but rather as increasing restriction of mobility, caused by the electric field. Whereas the action of the field on water in the macroscopic scale is obvious, such an action on the molecular scale is unlikely, since the local microscopic field strengths are orders of magnitude higher than the ones applied [31].

In any case, the $R$-variation of $v_{L}$ as derived from the inelastic peak width (Fig. 6) is inconsistent with a rather strong $T$-decrease associable with the sound velocity profile depicted in Fig. 5. A density decrease

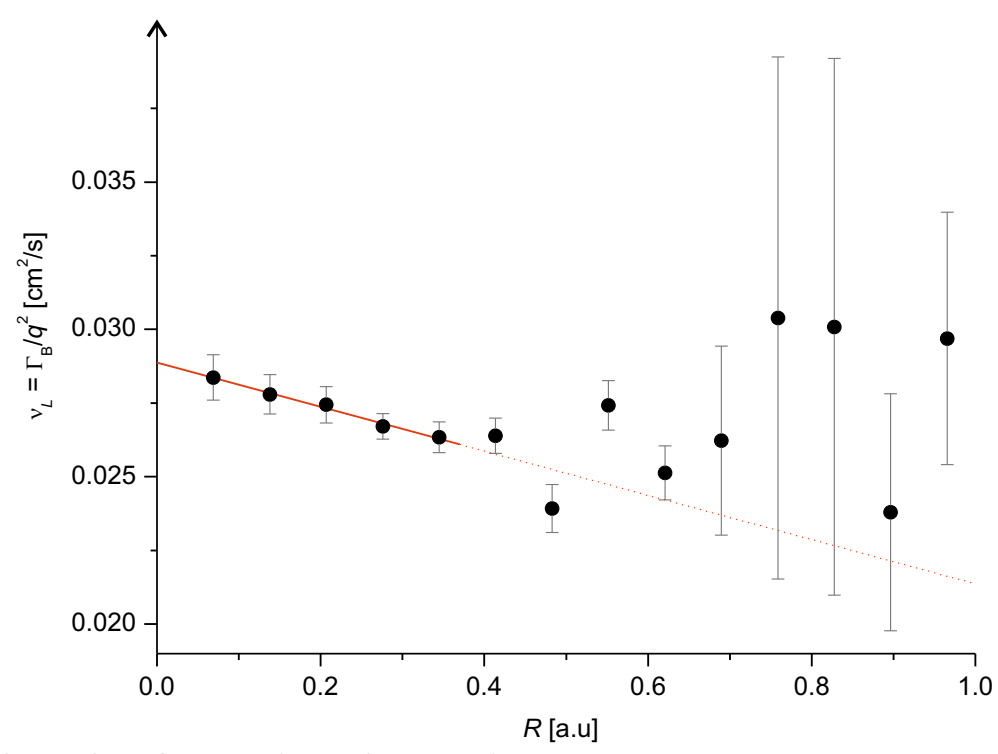

Fig. 6 Kinematic viscosity derived from the inelastic peak width.

The solid red line highlights the extrapolation of the viscosity at $R=0$ (the outer border of the bridge) from $R$ values up to $\sim 0.35$, which is consistent with the expected value for pure water at 1 bar and $46.1^{\circ} \mathrm{C}\left(\rho=989.8 \mathrm{kgm}^{-3}\right)$. The dotted line is an extrapolation towards higher $R$ values. 
due to nanometer sized bubbles in the bridge's interior would provide an alternative explanation for the narrowing peak-width. Without bubbles, one can assume that all illuminated portions of the bridge have the same weight in the final spectrum, resulting in an intensity increase, as observed from $R=0$ to $R=0.13$ (outer layer). The subsequent part of the spectrum can interpreted as a weighted average over a radial bubble distribution which causes attenuation by forward scattering at the bubbles in the bridge's interior. Such an attenuation can be observed for all three lines at $R$ values $>0.13$, with the elastic line showing an overlap of spurious scattering from the bridge's surface from $R$ $=0.6$ on. While keeping in mind that this is only a hypothesis since the actual density- (and thus the refractive index-) distribution within the bridge is unknown so far, it describes the observations well and thus depicts a possible scenario.

\subsection{Bubble Hypothesis}

Without bubbles on decreasing $T$ an increase of $v_{L}$ would be expected. As can be seen from Fig. 6, however, $v_{L}$ is more likely to decrease with $R$ instead. Such a $v_{L}$ decrease would exclude a strong $T$-decrease inside the bridge. Most remarkably, it would also imply that the $c$ and $v_{L}$ trends could not be concurrently accounted for by postulating that the bridge is made of bulk water at standard atmospheric pressure, emphasizing that Fig. 1 should be seen as simplified sketch. On the other hand, the $R=0$ extrapolated value of $v_{L}$, i.e. $v_{L}(R=0)=0.0289 \pm 0.0012 \mathrm{~cm}^{2} / \mathrm{s}$, is consistent with an external temperature of the bridge of $46{ }^{\circ} \mathrm{C}$, since we found that the ratio between longitudinal and shear viscosity is $4.9 \pm 0.2$, a value in fair agreement with previous determinations [32-34].

Earlier investigations [4, 5, 7] suggested the existence of micro- and nano- bubbles in the system. This assumption is corroborated by recent quantum field theory considerations [17], since the formation of coherence domains predicted therein would cause the exclusion of dissolved gasses, thus causing bubble formation. Hence, without excluding alternative explanations, such a "bubble plus water" system is postulated here and will be considered as adequate working hypothesis. In such a system the values of both $c$ and $v_{L}$ may be different from the ones of normal liquid water. Although these parameters are not a priori known, we assume that the density $(\rho)$ and $T$ dependence of $v_{L}$ follows the trend reported by Macedo and Litovitz [35]. This leads to the following expression for $v_{L} I / v_{L}(0)$ :

$\frac{v_{L}(R)}{v_{L}(0)} \propto \exp \left\{\frac{E_{a} \Delta T}{\mathrm{k}_{\mathrm{B}} T_{0} T}+\frac{V_{\min } \Delta \rho^{-1}}{\left(\rho^{-1}-V_{\min }\right)\left(\rho_{0}^{-1}-V_{\min }\right)}\right\}$

where $E_{a}$ is the activation energy (here assumed to be $17.5 \mathrm{~kJ} / \mathrm{mol}$ ), $\mathrm{k}_{\mathrm{B}}$ is the Boltzmann constant, $V_{\min }$ is the minimum volume allowed for the system, $T_{0}=46.1^{\circ} \mathrm{C}$ and $\rho_{0}=989.8 \mathrm{~kg} / \mathrm{m}^{3}$ is the water density at 1 bar and $46.1{ }^{\circ} \mathrm{C}$ [36]. Finally, $\Delta \rho^{-1}=\rho_{0}^{-1}-\rho^{-1}$ and $\Delta T=T_{0}-T$, where $\rho$ and $T$ are the density and temperature of the system at the actual $R$-value. According to Eq. (2) the decrease of viscosity with increasing $R$ can be due to a (rather large) temperature increase and/or to a density decrease. While an increase in $T$ above $46.1{ }^{\circ} \mathrm{C}$ is quite unlikely and inconsistent with sound velocity data, a marked $\rho$-decrease is in agreement with the hypothesis that the system is composed of pure water and bubbles, with larger bubble content inside the bridge.

Examples of density profiles obtained by employing Eq. (2) are reported as a function of $R$ in Fig. 7. For the forthcoming analysis, unless specified otherwise, an extrapolation of the data $R<0.5$ for the data $R>0.5$ was used. This procedure is motivated by the low inelastic signal and the concurrent growing up of an intense spurious elastic scattering coming from the reflection of UV beam by the back surface of the bridge (Fig. 1) observed at large $R$-values (Fig. 4). These effects strongly reduce the accuracy in the determination of $\Gamma_{\mathrm{B}}$.

$\mathrm{Up} /$ down triangles are data obtained considering $V_{\text {min }}=0.00085 / 0.00075 \mathrm{~m}^{3} \cdot \mathrm{kg}^{-1}$, and by setting $\Delta T=0$; i.e., by assuming that the $v_{L}$-variation is essentially due 


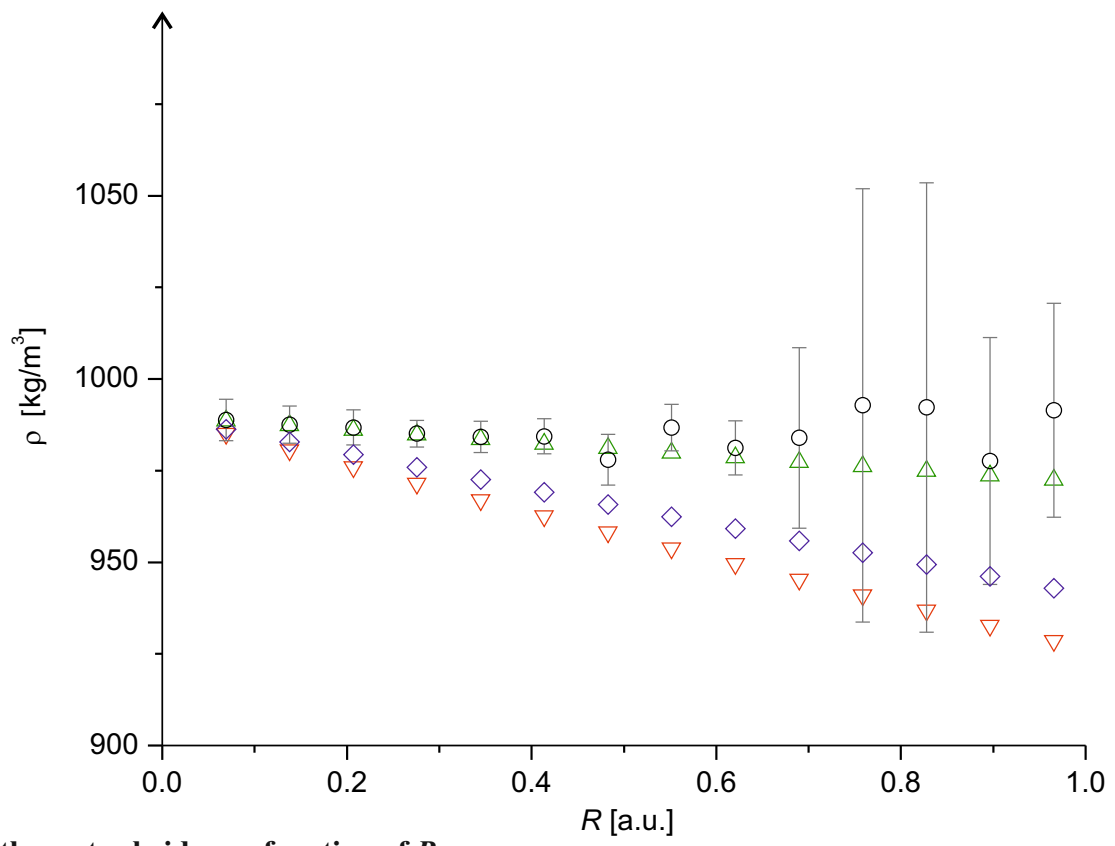

Fig. 7 Density of the water bridge as function of $\boldsymbol{R}$.

Up/down triangles are data obtained considering $V_{\text {min }}=0.00085 / 0.00075 \mathrm{~m}^{3} \cdot \mathrm{kg}^{-1}$; circles are data with $V_{\min }=0.00085 \mathrm{~m}^{3} \cdot \mathrm{kg}^{-1}$ but without extrapolation; diamonds correspond to data obtained setting $V_{\min }=0.00085 \mathrm{~m}^{3} \cdot \mathrm{kg}^{-1}$ and superposing a temperature gradient for the water surrounding the bubbles.

to a density change. Circles represent the data without extrapolation and considering $V_{\min }=0.00085 \mathrm{~m}^{3} \cdot \mathrm{kg}^{-1}$. Finally, diamonds correspond to data obtained by using the same minimum volume and assuming a constant temperature gradient: $\Delta T=\alpha_{T} R$, where $\alpha_{T}$ was treated as fitting parameter yielding $-21 \pm 12 \mathrm{~K} / R$. Although the assumptions we made concerning $V_{\min }$ and $E_{a}$ which are inherently affected by a certain degree of uncertainty, it is rather evident how the observed behavior of $v_{L}$ can be accounted for by postulating a strong $\rho$-decrease (eventually associated with a $T$-variation). Such a density change is not allowed for pure water, but it can surely occur in a heterogeneous system made of water and bubbles, thus supporting previous findings $[4,5,7]$.

Following this line of thought with the density of the bubble phase being much lower than that of water, it is straightforward to calculate the percentage of the bubble phase $\left(X_{b u b}=100 \cdot\left(\rho_{0}-\rho\right) / \rho_{0}\right)$ as a function of $R$. The results are given in Fig. 8. Moreover, by joining sound velocity data and by assuming that the contributions of the bubbles and of surrounding water simply add one to the other, one can also estimate the compressibility of the bubble subsystem: $\chi_{s, b u b}=\left(M_{w^{\prime}}-\left(1-X_{b u b}\right) M_{B}\right) /\left(X_{b u b} M_{W} M_{B}\right)$, where $M_{B}=\rho c^{2}$ and $M_{w}=\rho_{w} c_{w}{ }^{2}$ are the bulk moduli of the system and of the surrounding water, respectively, $\rho_{w}$ and $c_{w}{ }^{2}$ being the density and sound velocity of pure water at the corresponding $(\rho, T)$-value, respectively [36]. The results of these calculations are reported in Figs. 9 and 10 , respectively.

\section{Discussion}

\subsection{Bubbles and Molecular Mobility}

The inelastic UV scattering of an aqueous electrohydrodynamic floating bridge reveals a variety of features: With increasing laser penetration, Stokes and Anti-Stokes lines are inclined, their broadness first in- and then decreases (Fig. 2). An interpretation is not straight forward, since these behaviors illustrate contradictory density and temperature trends of the bulk water the bridge is supposed to be made of. The contradictions disappear if second, less dense phase is postulated: Bubbles.

Alternatively, the gradient in sound velocity could be interpreted as a gradient in molecule mobility. This 


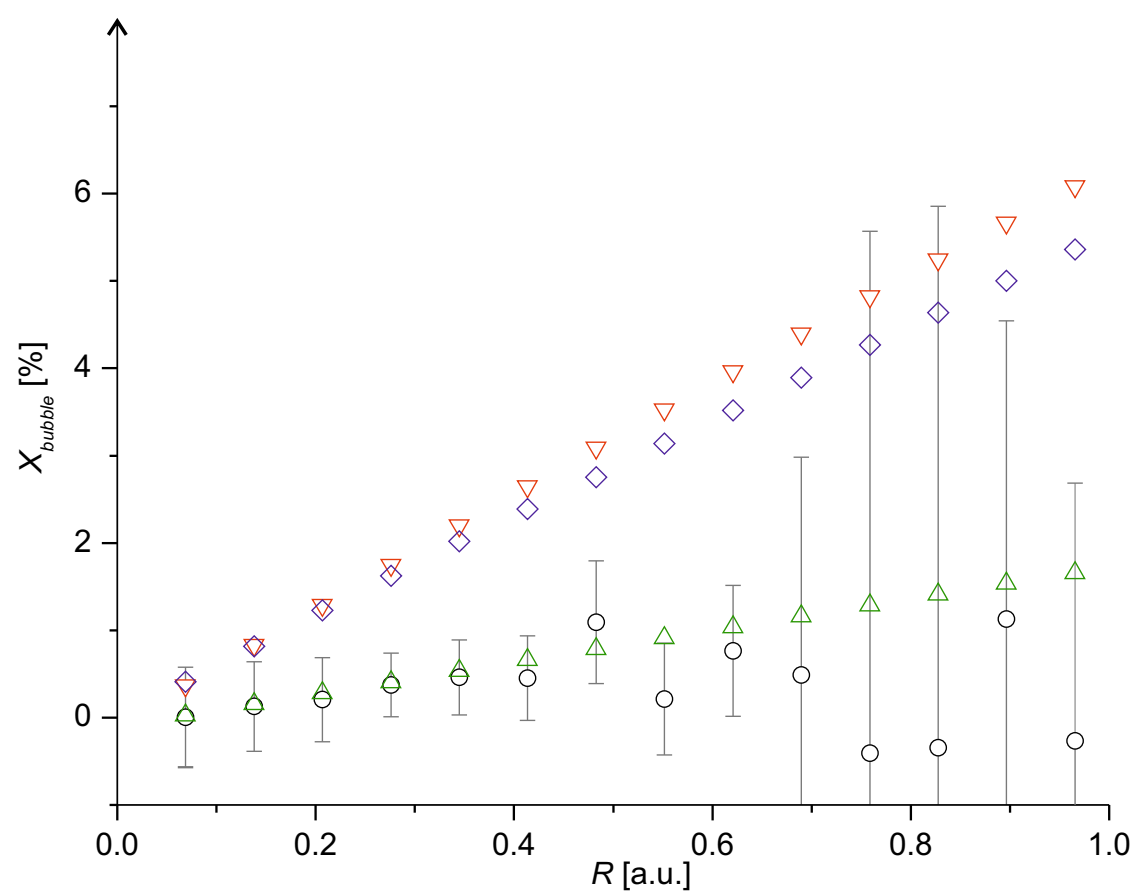

Fig. 8 The fraction of bubbles determined from the data reported in Fig. 7.

Up/down triangles are data obtained considering $V_{\text {min }}=0.00085 / 0.00075 \mathrm{~m}^{3} \cdot \mathrm{kg}^{-1}$; circles are data with $V_{\text {min }}=0.00085 \mathrm{~m}^{3} \cdot \mathrm{kg}^{-1} \mathrm{but}$ without extrapolation; diamonds correspond to data obtained setting $V_{\min }=0.00085 \mathrm{~m}^{3} \cdot \mathrm{kg}^{-1}$ and superposing a temperature gradient for the water surrounding the bubbles.

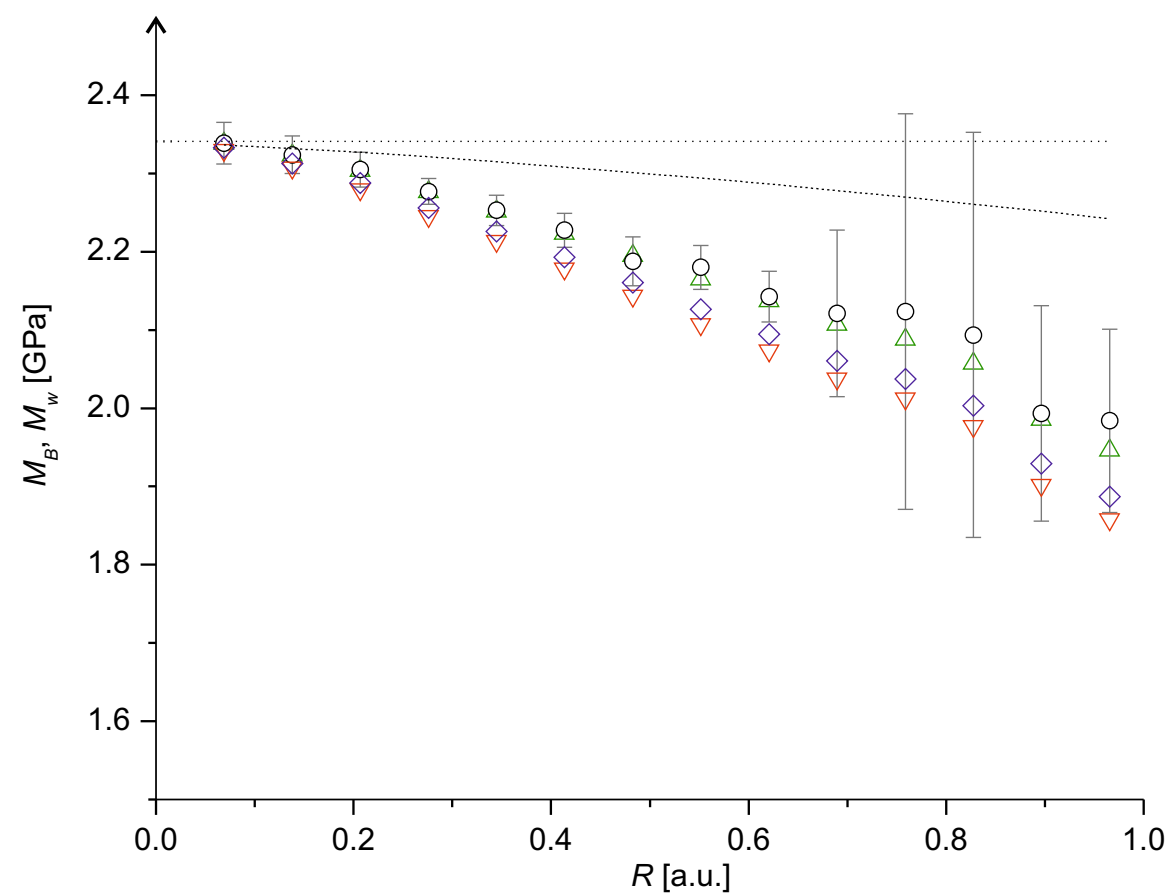

Fig. 9 Bulk modulus of the water bridge and of water subsystem.

The dashed horizontal line shows the modulus of water at $\mathrm{T}=46.1^{\circ} \mathrm{C}$, the dotted line below includes a temperature gradient. Up/down triangles are data obtained considering $\mathrm{V}_{\text {min }}=0.00085 / 0.00075 \mathrm{~m}^{3} \cdot \mathrm{kg}^{-1}$; circles are data with $\mathrm{V}_{\text {min }}=0.00085 \mathrm{~m}^{3} \cdot \mathrm{kg}^{-1}$ but without extrapolation; diamonds correspond to data obtained setting $\mathrm{V}_{\min }=0.00085 \mathrm{~m}^{3} \cdot \mathrm{kg}^{-1}$ and superposing a temperature gradient for the water surrounding the bubbles. 


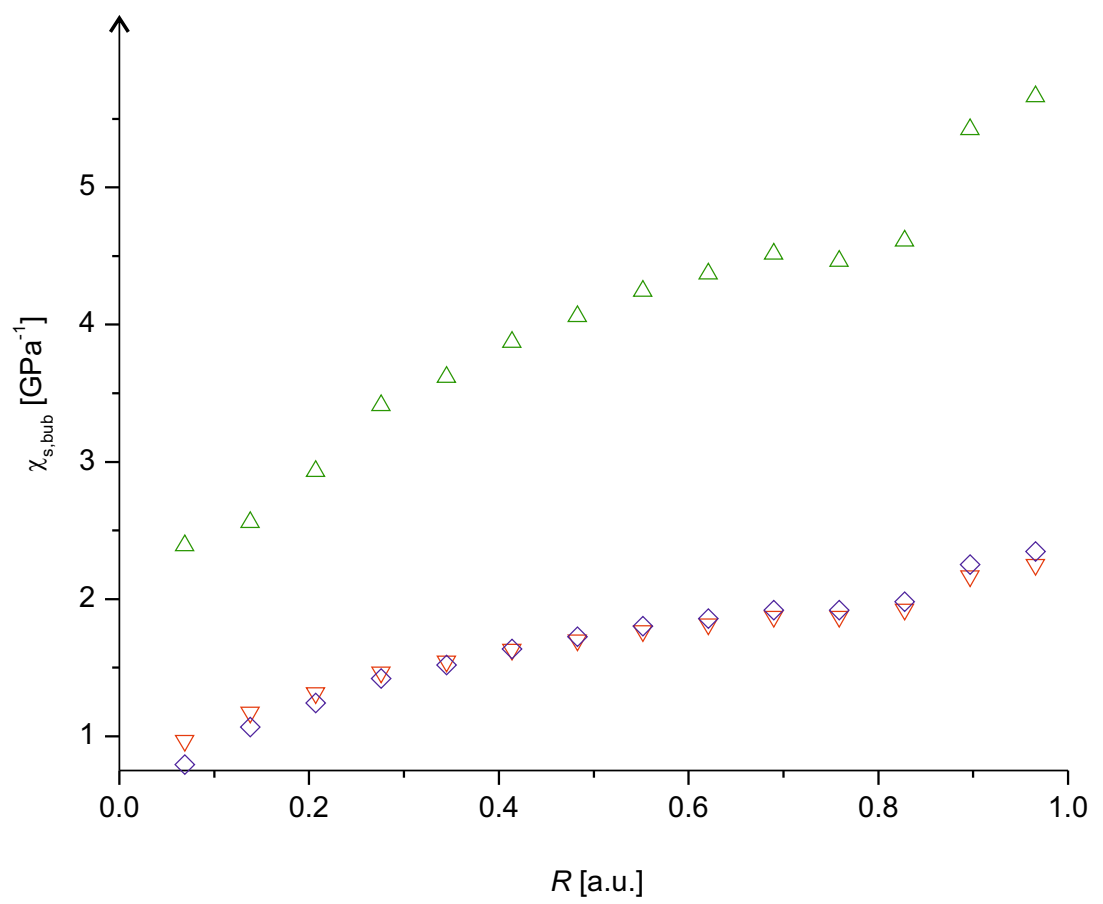

Fig. 10 Compressibility of the bubbles inside the water bridge derived from data reported in Fig. 9.

$\mathrm{Up} /$ down triangles are data obtained considering $V_{\min }=0.00085 / 0.00075 \mathrm{~m}^{3} \cdot \mathrm{kg}^{-1}$; diamonds correspond to data obtained setting $V_{\min }=$ $0.00085 \mathrm{~m}^{3} \cdot \mathrm{kg}^{-1}$ and superposing a temperature gradient for the water surrounding the bubbles.

could be attributed to coherence-domains which are predicted to form within the bridge [17].

Whereas there is little evidence for the existence of such domains, the introduction of a bubble phase seems reasonable since optical measurements $[4,7]$ neutron scattering [5] and related investigations [37] show that an electric field can cause the formation of nano- and micro-sized bubbles.

Since no diffuse scattering is observed, the portion of the bridge crossed by the UV beam is homogeneous over length scales larger than $2 \pi / Q$. Therefore, the estimated size of the bubbles should roughly be $\leq 100$ $\mathrm{nm}$. This assumption is supported by the deduced bubble compressibility (Fig. 10) which is very low compared to the compressibility of normal gasses, a behavior consistent with suggested properties of nano bubbles [42]. They are supposedly concentrated in the middle of the bridge, since in the outer shell both sound velocity and viscosity match the values of bulk water at about $46{ }^{\circ} \mathrm{C}$, a temperature in agreement with thermographic investigations [7].

In view of the fact that at large $R$-values we are dealing with an average of sample properties that extends almost over the whole bridge diameter, one can suppose that the actual values of the derived physical quantities showed in Figs. 7-10 present even larger deviations in the bridge core with respect to the outer layers (i.e., for $R \sim 0$ ). If we assume that, e.g., $3 \%$ of the bridge volume is occupied by bubbles (Fig. 8) of $50 \mathrm{~nm}$ radius, one can deduce a mean numerical density of 57 bubbles $/ \mu \mathrm{m}^{3}$ or to a mean bubble distance of $\sim 260$ $\mathrm{nm}$ if, e.g., a cubical distribution of bubbles is assumed.

\subsection{Exclusion Zone Water}

We would like to make a cross reference here to the so-called "exclusion zone" (EZ) water, an issue thoroughly discussed in older Refs. [38, 39] and more recent literature [40, 41]. Zheng et al. [41] report that this special water absorbs UV light at $270 \mathrm{~nm}$ with a FWHM of 50-100 nm depending on the distance from a Nafion surface. This absorption could explain the attenuation of the inelastic beams (Fig. 2 and the discussion in the results section). EZ water is said to appear close to highly charged surfaces (like Nafion), having, in contrast to bulk water, long range ordering. Similar to a charged surface, a strong electric field and 
considerable electrical current flow are present in this experiment, and an indication of long range ordering inside the water bridge was given by $2 \mathrm{D}$ neutron scattering [6]. Therefore, we suggest many effects reported as "EZ water" can be compared to the ones reported in this paper, and the underlying physics are most probably the same.

\subsection{Kinematic Viscosity and Minimal Volume}

In Fig. 6, the longitudinal kinematic viscosity derived from the inelastic peak width is shown. In homogeneous systems, the viscosity depends on temperature and density. Whereas the temperature dependence follows an activation law, the density dependence is mainly due to the reduction of the free volume [35], which means that the viscosity increases both at low temperature and high density. However, within the water bridge, the average viscosity decreases with laser penetration depth, indicating a lower density and/or a higher temperature within the bridge. If the results of Macedo and Litovitz [35] are used to calculate the density profile on a two-phase system, the leading effect responsible for the observed decrease of viscosity is the free volume reduction. This explains the seeming contradiction between the inclination of the scattered lines (temperature decrease) and the viscosity trend (temperature increase). Yet, a further comment to this assumption should be made. Although the values of minimum volume and activation energy chosen for our system are reasonable, the results presented are very sensitive with respect to this choice. Therefore, the authors plan to conduct additional experiments, also using different scattering angles, in order to map the entire cross section of the water bridge. This would allow using the minimum volume (which is the most sensitive parameter) as fitting parameter.

\section{Conclusions}

The first IUVS scattering measurements of a floating EHD bridge can be summarized as follows: Stokes and Anti-Stokes lines are inclined when plotted against laser penetration depths; their intensity first in-, and then decreases. From the inclination a gradient in average sound velocity can be calculated; all observed features can be satisfactorily explained by the introduction of previously proposed nano bubbles. The interior of the bridge exhibits a low compressibility which is consistent with proposed nano bubbles behavior [42]. The existence of micro and nano bubbles in water has been described before in the framework of the application of an electric field on a nanoscale water-based lubricating film [42], laser-induced cavitation [43], radio frequency treatment [44], and biofilms [45].

Alternatively, a gradient in molecular mobility introduced by the action of the electric field on the water dipoles could account for the effects observed.

\section{Acknowledgments}

This work was performed in the TTIW-cooperation framework of Wetsus, Centre of Excellence for Sustainable Water Technology (www.wetsus.nl). Wetsus is funded by the Dutch Ministry of Economic Affairs, the European Union Regional Development Fund, the Province of Fryslân, the City of Leeuwarden and the EZ/Kompas program of the "Samenwerkingsverband Noord-Nederland". The authors would like to thank Marie-Claire Bellissent-Funel (Laboratoire Léon Brillouin, Saclay), Eshel Ben-Jacob (Tel Aviv University), Mariano Bizzarri (Università La Sapienza, Roma), Harry Bruning (Wetsus), Cees Buisman (Wetsus), Gert-Jan Euverink (Wetsus), Friedemann Freund (NASA SETI Institute, California), Karl Gatterer (Graz University of Technology), Emilio Del Giudice (Universitá di Milano), Ferenc Hajdu (Central Research Institute for Chemistry, Hungarian Academy of Sciences, Budapest), Franz Heitmeir (Graz University of Technology), Jan C. M. Marijnissen (Delft University of Technology), Hideo Nishiumi (Chem. Eng. Lab., Hosei University, Japan), Laurence Noirez (Laboratoire Léon Brillouin, CEA-CNRS/IRAMIS, 
CEA-Saclay), Gerald H. Pollack (University of Washington), Alan Soper (Rutherford Appleton Laboratories, ISIS, Oxford), Piergiorgio Spaggiari (Istituti Ospitalieri Di Cremona, Milano, Italy), José Teixeira (Laboratoire Léon Brillouin, CEA-CNRS/IRAMIS, CEA/Saclay), Giuseppe Vitiello (Universitá degli studi di Salerno), Vladimir Voeikov (M.V. Lomonosov Moscow State University), as well as Luewton L.F. Agostinho, Cees Kamp, Ingo Leusbrock, Astrid H. Paulitsch-Fuchs, Martina Sammer and Adam D. Wexler (Wetsus) for the ongoing discussion on the water bridge phenomenon (in alphabetic order). The financial support of the participating companies of the Applied Water Physics Theme at Wetsus, Centre of Excellence for Sustainable Water Technology, is acknowledged.

\section{References}

[1] W.G. Armstrong, Electrical Phenomena, The Electrical Engineer 1893, pp. 145-154.

[2] W. Uhlig, Personal Communication, Laboratory of Inorganic Chemistry, ETH Hönggerberg-HCI, CH-8093 Zürich, 2005.

[3] E.C. Fuchs, J. Woisetschläger, K. Gatterer, E. Maier, R. Pecnik, G. Holler, et al., The floating water bridge, J. Phys. D: Appl. Phys. 40 (2007) 6112-6114.

[4] E.C. Fuchs, K. Gatterer, G. Holler, J. Woisetschläger, Dynamics of the floating water bridge, J. Phys. D: Appl. Phys. 41 (2008) 185502-185507.

[5] E.C. Fuchs, B. Bitschnau, J. Woisetschläger, E. Maier, B. Beuneu, J. Teixeira, Neutron scattering of a floating heavy water bridge, J. Phys. D: Appl. Phys. 42 (2009) 065502:1-065502:4.

[6] E.C. Fuchs, P. Baroni, B. Bitschnau, L. Noirez, Two-dimensional neutron scattering in a floating heavy water bridge, J. Phys. D: Appl. Phys. 43 (2010) 105502:1-105502:5.

[7] J. Woisetschläger, K. Gatterer, E.C. Fuchs, Experiments in a floating water bridge, Exp. Fluids 48-1 (2010) 121-131.

[8] E.C. Fuchs, L.L.F. Agostinho, M. Eisenhut, J. Woisetschläger, Mass and charge transfer within a floating water bridge, Proc. SPIE 2010, 7376, 73761E1-15.

[9] A. Castellanos, Electrohydrodynamics: No. 380 (Courses and Lectures), Springer, Vienna, Austria, 1998.

[10] J. Mrázek, J.V. Burda, Can the $\mathrm{pH}$ value of water solutions be estimated by quantum chemical calculations of small water clusters, J. Chem. Phys. 125 (2006) 194518:1-194518:14

[11] W.L. Jorgensen, J. Tirado-Rives, Potential energy functions for atomic-level simulations of water and organic and biomolecular systems, PNAS 102 (2005) 6665-6670.

[12] E. Del Giudice, Old and new views on the structure of matter and the special case of living matter, J. Phys.: Conf. Ser. 67 (2006) 012006.

[13] T. Head-Gordon, M.E. Johnson, Tetrahedral structure or chains for liquid water, PNAS 21 (2006) 7973-7977.

[14] H.E. Stanley, S.V. Buldyrev, G. Franzese, N. Giovambattista, F.W. Starr, Static and dynamic heterogeneitities in water, Phil. Trans. R. Soc. A 363 (2005) 509-523.

[15] C.A. Chatzidimitriou-Dreismann, T.A. Redah, R.M.F. Streffer, J. Mayers, Anomaleous deep inelastic neutron scattering from liquid $\mathrm{H}_{2} \mathrm{O}-\mathrm{D}_{2} \mathrm{O}$ : Evidence of nuclear quantum entanglement, Phys. Rev. Lett. 79 (1997) 2839-2842.

[16] R. Arani, I. Bono, E. Del Giudice, G. Preparata, QED coherence and the thermodynamics of water, Int. J. Mod. Phys. B 9 (1995) 1813-1841.

[17] E. Giudice, E.C. Fuchs, G. Vitiello, Collective molecular dynamics of a floating water bridge, Water (Seattle) 2 (2010) 69-82.

[18] R.C. Ponterio, M. Pochylski, F. Aliotta, C. Vasi, M.E. Fontanella, F. Saija, Raman scattering measurements on a floating water bridge, J. Phys. D: Appl. Phys. 43 (2010) 175405:1-175405:8.

[19] H. Nishiumi, F. Honda, Effects of electrolyte of floating water bridge, Res. Let. Phys. Chem. (2009) 371650:1-371650:3.

[20] M. Eisenhut, X. Guo, A.H. Paulitsch-Fuchs, E.C. Fuchs, Aqueous phenol and ethylene glycol solutions in electrohydrodynamic liquid bridging, Cent. Eur. J. Chem. 9 (2011) 391-403.

[21] E.C. Fuchs, L.L.F. Agostinho, A. Wexler, R.M. Wagterveld, J. Tuinstra, J. Woisetschläger, The behaviour of a floating water bridge under reduced gravity conditions, J. Phys. D: Appl. Phys. 44 (2011) 025501:1-025501:8.

[22] A.G. Marin, D. Lohse, Building water bridges in air: Electrohydrodynamics of the floating water bridge, Phys. Fluids 22 (2010) 122104:1-122104:9.

[23] A. Widom, J. Swain, J. Silverberg, S. Sivasubramanian, Y.N. Srivastava, Theory of the Maxwell pressure tensor and the tension in a water bridge, Phys. Rev. E 80 (2009) 016301:1-016301:7.

[24] A.A. Aerov, Why the water brid ge does not collapse, Phys. Rev. E 84 (2011) 036314:1-036314:4.

[25] E.C. Fuchs, Can a century old experiment reveal hidden 
properties of water, Water (MDPI) 2 (2010) 381-410.

[26] G.H. Pollack, Cells, Gels and the Engine of Life, Ebener \& Sons: Seattle WA, 2001.

[27] C. Masciovecchio, F. Bencivenga, A. Gessini, Water dynamics at the nanoscale, Condens. Matt. Phys. 11 (2008) 47-56.

[28] B.J. Berne, R. Pecora, Dynamic Light Scattering, John Wiley \& Son, New York, 1975.

[29] G. Ruocco, F. Sette, The high frequency dynamics of liquid water, J. Phys.: Condens. Matter 11 (1999) R259-R293.

[30] S.C. Santucci, D. Fioretto, L. Comez, A. Gessini, C. Masciovecchio, Is there any fast sound in water, Phys. Rev. Lett. 97 (2006) 225701:1-225701:4.

[31] D. Rai, A. D. Kulkami, S.P. Gejji, R.K. Pathak, Water clusters $\left(\mathrm{H}_{2} \mathrm{O}\right)_{\mathrm{n}}, \mathrm{n}=6-8$, in external electric fields, J. Chem. Phys. 128 (2008) 034310:1-034310:14.

[32] J.M.M. Pinkerton, A pulse method for the measurement of ultrasonic absoprtion in liquids: Results for water, Nature 160 (1947) 128-129.

[33] J. Rouch, G.C. Lai, S.H. Chen, Brillouin scattering studies of normal and supercooled water, J. Chem. Phys. 65 (1976) 4016-4021.

[34] A. Cunsolo, M. Nardone, Velocity dispersion and viscous relaxation in supercooled water, J. Chem. Phys. 105 (1996) 3911-3917.

[35] P.B. Macedo, T.A. Litovitz, On the relative roles of free volume and activation energy in the viscosity of liquids, J. Chem. Phys. 42 (1965) 245-256.

[36] W. Wagner, A. Pruss, The IAPWS formulation 1995 for the thermodynamic properties of ordinary water substance for general and scientific use, J. Phys. Chem. Ref. Data 31 (2002) 387-535.

[37] G. Xie, J. Luo, S. Liu, C. Zhang, X. Lu, Micro-bubble phenomenon in nanoscale water-based lubricating film induced by external electric field, Tribol. Lett. 29 (2008) 169-176.

[38] J.C. Henniker, The depth of the surface zone of a liquid, Rev. Mod. Phys. 21 (1949) 322-341.

[39] A. Szent-Gyorgyi, Bioenergetics, Acad. Press, New York, 1957.

[40] G.N. Ling, In Search of the Physical Basis of Life, Plenum, New York, 1984.

[41] J.M. Zheng, W.C. Chin, E. Khijniak, G.H. Pollack, Surfaces and interfacial water: evidence that hydrophilic surfaces have long-range impact, Adv. Coll. Interf. Sci. 127 (2006) 19-27.

[42] F. Jin, J. Ye, L. Hong, H. Lam, C. Wu, Slow relaxation mode in mixtures of water and organic molecules: Supramolecular structures or nanobubbles, J. Phys. Chem. B 111 (2007) 2255-2261.

[43] X. M. Liu, J. He, J. Lu, X.W. Ni, Growth and collapse of laser-induced bubbles in glycerol-water mixtures, Chin. Phys. B 17 (2008) 2574-2579.

[44] Y. Katsir, L. Miller, Y. Aharanov, E.B. Jacob, The effect of rf-irradiation on electrochemical deposition and its stabilization by nanoparticle doping, J. Electrochem. Soc. 154 (2007) D249-D259.

[45] E.B. Jacob,Y. Aharonov, Y. Sh apira, Bacteria harnessing complexity, Biofilms 1 (2004) 239-263. 